\title{
The Impacts of Node Distribution on the Effective Transport Capacity of Ad Hoc Dispersed Spectrum Cognitive Radio Networks
}

\author{
Khalid. A. Qaraqe ${ }^{\mp}$, Muneer Mohammad ${ }^{*}$, Hasari Celebi ${ }^{\mp}$, Amer El-Saigh $^{\#}$ \\ ₹ Electrical and Computer Eng. Dept., Texas A\&M University at Qatar, P.O. Box 23874, Doha, Qatar \\ * Electrical and Computer Eng. Dept., Texas A\&M University, College Station, Texas, 77843, USA \\ \#Networks R\&D, Qatar Telecom (Qtel) Q.S.C., P.O. Box 217, Doha, Qatar \\ Emails: \{khalid.qaraqe, hasari.celebi\}@qatar.tamu.edu,m.muneer@tamu.edu, aelsaigh@qtel.com.qa
}

\begin{abstract}
Cognitive radio networks that employ the dispersed spectrum utilization as spectrum access method are called dispersed spectrum cognitive radio networks. In this paper, we derive the effective transport capacity of adhoc dispersed spectrum cognitive radio networks in which the nodes are distributed in three dimensional (3D) configurations. The analysis is performed considering a cubic grid distribution for the node configuration and inter-node interference (INI). Numerical results are provided to show the effects of node distribution, fading, and modulation on the effective transport capacity of adhoc dispersed spectrum cognitive radio networks.
\end{abstract}

Keywords; Wireless adhoc, cognitive radio, effective transport capacity, Nakagami-m fading.

\section{INTRODUCTION}

Wireless adhoc networks are attractive due to their unique advantages such as that they can be deployed without requiring any pre-existing infrastructure [1]. Wireless adhoc networks neither have fixed topology nor centralized servers; once an adhoc network is deployed, the network nodes operate autonomously to form a communications network and provide connectivity to the destination to deliver the data throughout hops with an acceptable bit error rate (BER) [1].

In [2], 2D and 3D structures for underwater sensor networks are proposed where the main objective was to determine the minimum number of sensors and redundant sensor nodes for achieving communication coverage. In [3], the effect of nonuniform random node distribution on the throughput of Medium Access Protocol (MAC) is investigated through simulation without providing theoretical analysis. In [4], the problem of efficient geometric routing in 3D networks has been addressed. Additionally, several studies in the literature investigated the use of location information in order to enhance the performance of cognitive radio networks, e.g., [5, 6]. The conclusion drawn is that use of network topology information could bring significant benefits to cognitive radios and networks to reduce the maximum transmission power and spectral impact of the topology [7]. In [8, 9, 11], a new communication-theoretic approach to the analysis of adhoc networks is proposed. The concept of effective transport capacity that quantifies the actual bandwidth-distance product supported by the network is introduced in [9]. In [11], the effects of modulation formats on the effective transport capacity of 2D adhoc wireless networks are investigated. The performance analysis is conducted over both AWGN and Rayleigh channels in the absence and presence of inter-node interference (INI). In addition, RESGO and RESCHOGO MAC protocols are considered during the analysis.

The previous works in the conventional adhoc wireless networks lack to provide a practical and comprehensive effective transport capacity performance analysis considering 3D node distributions. In addition, there is not any study in the literature that provides such performance analysis for adhoc cognitive radio networks to the best of our knowledge. Hence, the contributions of this paper can be summarized as follows. In this paper, a practical and comprehensive effective transport capacity based performance analysis in adhoc dispersed spectrum cognitive radio networks considering $3 \mathrm{D}$ node distribution is presented. Note that dispersed spectrum cognitive radio is a novel cognitive radio system that utilizes frequency diversity techniques [10]. In other words, the signal is replicated $K$ times (number of available bands that are used opportunistically) to create frequency diversity. Then, these $K$ signal copies are transmitted over $K$ number of available bands (i.e. channels), received and combined using diversity combining methods. As a result, in this paper, two nodes in the adhoc dispersed spectrum cognitive radio networks communicate over $\mathrm{K}$ number of bands (i.e. channels), which differentiate our works from all the previous works. Furthermore, the performance analysis of adhoc dispersed spectrum cognitive radio networks is conducted when the $K$ bands experience independent and dependent Nakagami-m fading. 
This paper is organized as follows. In section II, the system model is described. In Section III, the analysis of the effective transport capacity for the $3 \mathrm{D}$ is provided. In section IV, some numerical results are presented and Section $\mathrm{V}$ provides the conclusions.

\section{SYSTEM MODEL}

The network communications system model considered in this study is summarized by the following features $[8,9,11]$ :

- Each node transmits a fixed power of $60 \mu w$ and the multi-hop routes between a source and destination is established by a sequence of minimum length links. Moreover, no node can share more than one route.

- If a node needs to communicate with another node, a multi hop route is first reserved and only then the packets can be transmitted without looking at the status of the channel which is based on MAC protocol for Inter-Node Interference (INI): Reserve and go (RESGO) [11]. Packet generation, with each packet having a fixed length of $L$ bits, is given by a Poisson process with parameter $\lambda$ (packets/second).

- The INI experienced by the nodes in the network is mainly dependent on the node distribution and the MAC protocol.

- The condition $\lambda L \leq R_{b}$, where $R_{b}$ is transmission data rate of the nodes, needs to be satisfied for network communications.

In the 3D node configuration, there are $N$ nodes and each node is placed uniformly at the center of a cubic grid in a spherical volume $V$ that can be defined as

$V \approx N d_{l}^{3}$

where $d_{l}$ is the length of cube that a node is centered in. From (1), it can be shown that two neighboring nodes are at distance $d_{l}$, which is defined as

$d_{l} \approx\left(1 / \rho_{s}\right)^{\frac{1}{3}}$

where $\rho_{s}=N / V$ (unit $\mathrm{m}^{-3}$ ) is the node volume density. The maximum number of hops $\left(n_{h}^{\max }\right)$ must be determined first in order to determine the average number of hops, $\left(\bar{n}_{h}\right)$. The deviation from a straight line between the source and destination nodes is limited by assuming that the source and destination nodes lie at opposite ends of a diameter over a spherical surface and a large number of nodes in the network volume are simulated [8]. It follows that $n_{h}^{\max }$ distribution can be defined for $3 \mathrm{D}$ configuration as

$$
n_{h}^{\max }=\left\lfloor\frac{\text { diameter }_{\text {surf ace }}}{d_{l}}\right\rfloor=\left\lfloor 2\left(\frac{3 N}{4 \pi}\right)^{1 / 3}\right\rfloor,
$$

where \lfloor\rfloor indicates the integer value closest to the argument. Since the number of hops is assumed to have a uniform distribution, the probability density function (PDF) can be defined as

$$
P_{n_{h}}(x)=\left\{\begin{array}{cc}
\frac{1}{n_{h}^{\max }}, & 0<x<n_{h}^{\max } \\
0, & \text { otherwise }
\end{array}\right.
$$

therefore,

$\bar{n}_{h}=\int_{0}^{n_{h}^{\max }} \frac{1}{n_{h}^{\max }} x d x=\frac{n_{h}^{\max }}{2}$,

which is the same result derived in [8]. The average number of hops for $3 \mathrm{D}$ configuration can therefore be obtained as

$\overline{n_{h}}=\left\lfloor\left(\frac{3 N}{4 \pi}\right)^{1 / 3}\right\rfloor$

\section{ANALYSIS OF EFFECTIVE TRANSPORT CAPACITY}

The effective transport capacity performance analysis of conventional adhoc wireless networks considering 2D node distribution is conducted in [11]. In section, this analysis extended to adhoc dispersed spectrum cognitive radio networks [10] where the nodes are distributed in 3D. The total effective transport capacity, $\left(C_{T}\right)$, is the summation of effective transport capacity for each route. Since the routes are disjoint, the $C_{T}$ is derived in [9] as

$$
C_{T}=\lambda L \bar{n}_{s h} d_{l} N_{a r}
$$

where $N_{a r}$ is the number of disjoint routes and $\bar{n}_{s h}$ is the average number of sustainable hops [9] which is defined as

$$
\bar{n}_{s h}=\min \left\{\sum_{s h}^{\max }, \bar{n}_{h}\right\}=\min \left\{\left\lfloor\frac{\ln \left(1-B E R^{\max }\right)}{\ln \left(1-B E R_{L}\right)}\right\rfloor, \bar{n}_{h}\right\},
$$

where $B E R_{L}$ and $B E R^{\max }$ are the BER at the end of a single link and the maximum $B E R$ can be tolerated to receive the 
data, respectively. The average $B E R$ at the end of a multi-hop route can therefore be expressed in [9] as

$$
\overline{B E R}=B E R^{\left(\bar{n}_{h}\right)}=1-\left(1-B E R_{L}\right)^{\bar{n}_{h}} .
$$

In this paper, we focus on M-QAM modulation for the independent and dependent fading cases in order to determine the $B E R_{L}$. In general, the BER can be obtained by averaging the bit error probability for AWGN as in [12] over Nakagami$\mathrm{m}$ fading distribution channel, which is given in [13] by

$$
\begin{aligned}
& B E R_{L}=\int_{0}^{\infty} P_{e}(\gamma) P_{\gamma}(\gamma) d \gamma \\
& \quad=\frac{4}{\pi \log _{2}(M)}\left[\left(1-\frac{1}{\sqrt{M}}\right) \int_{0}^{\pi / 2} \mu_{\gamma s}(s) d \phi-\left(1-\frac{1}{\sqrt{M}}\right) \int_{0}^{2} \mu_{\gamma s}(s) d \phi\right]
\end{aligned}
$$

where $\boldsymbol{\mu}_{\gamma s}$ is the moment generating function (MGF) and it is defined as the Laplace transform of the PDF of the output $S N R_{L}, s=\frac{-g}{\sin \phi^{2}}, g$ is a function of the size of M-QAM and it is defined as $g=\frac{1.5}{M-1}$.

Unlike to the adhoc wireless network model in the literature, in the adhoc dispersed spectrum cognitive radio networks, the total $S N R_{L}$ for $i=1,2,3 . . K$ dispersed bands at each node is linear sum of SNR that are collected at $K$ bands, which can be expressed as

$$
S N R_{L}=\sum_{i=1}^{K} S N R_{i}
$$

The $S N R_{L}$ for the adhoc dispersed spectrum cognitive radio networks can be expanded, as in [11] to be written in the form of SNR of $i^{\text {th }}$ band with respect to the SNR of the first band. Hence, assume that the received power from the first band is equal to $P$ and the AWGN experienced in this band has a power spectral density of $N_{0}$. Assuming that the received power from the $i^{t h}$ band is equal to $\left(\delta_{i} p\right)$ and the AWGN experienced in this band has a power spectral density of $\left(\beta_{i} N_{0}\right)$. Thus, the total $S N R_{L}$ at each node in adhoc dispersed spectrum cognitive radio networks can be expressed as

$$
S N R_{L}=\sum_{i=1}^{K} \alpha^{2} \kappa_{i} \frac{\mu P_{t} / d_{l}^{2}}{F K T_{0} R_{b}+P_{I N l} \eta}
$$

where $\kappa_{i}=\frac{\delta_{i}}{\beta_{i}}, \kappa_{1}=1, P_{t}$ is the transmitted power from each node, $F$ is the noise figure and $K=1.38 \times 10^{-23} \mathrm{~J} / \mathrm{K}$ is the Boltzmann's constant, $T=300 K$ is the room temperature, $\alpha$ is the fading envelope, where $\eta$ is the spectral efficiency, $P_{I N I}$ is the INI power, and $\mu$ can be expressed as

$$
\mu=\frac{G_{t} G_{r} c^{2}}{(4 \pi)^{2} f_{l} f_{c}^{2}}
$$

where $G_{t}$ and $G_{r}$ are the transmitter and receiver antenna gains, respectively, $f_{c}$ is the carrier frequency, $c=3 \times 10^{8} \mathrm{~m} / \mathrm{s}$ is the speed of light, and $f_{l} \leq 1$ is the loss factor which is defined as the losses caused by the hardware devices.

For independent Nakagami-m fading channels, it is assumed that each of the $K$ band channels has Nakagami-m distribution, which implies that an instantaneous $S N R$ for each band will have gamma distribution. Therefore, the MGF is defined in [14] and can be written in terms of the $S N R_{L}$ here. Thus, the MGF takes the following form,

$$
\mu_{\gamma s}(s)=\left(1-\frac{s \log _{2}(M) S N R_{L}\left(\kappa_{i}\right)}{m \sum_{i=i}^{K} \kappa_{i}}\right)^{-m k} .
$$

For dependent Nakagami-m fading channels, it is assumed that $K$ bands are correlated, each having Nakagami-m distribution. The basic idea is to try to express the $S N R$ in terms of Gaussian distribution, since it is a simple approach to deal with Gaussian distribution regardless of the complexity. The instantaneous $S N R$ of parameter $m_{i}$ for each band can be considered as the sum of squares of $2 m_{i}$ independent Gaussian random variables (RVs) which mean that the covariance matrix of the total $S N R_{L}$ can be expressed by $\left(2 \sum_{k=1}^{K} m_{i}\right) \times\left(2 \sum_{k=1}^{K} m_{i}\right)$ matrix with correlation coefficient between Gaussian RVs [15]. We use two correlation matrices, as in [16] for the sake of analysis, which are for linear and triangular arrays and these two matrices are referred as Configuration $\mathrm{A}$ and Configuration $\mathrm{B}$, respectively, in this study.

The MGF of Nakagami-m fading for the dependent case is defined as [17]

$$
\mu_{\gamma s}(s)=\frac{1}{\prod_{n=1}^{N}\left(1-2 s \rho_{n}\right)^{\frac{1}{2}}},
$$

where $N=2 \sum_{i=1}^{K} m_{i}$ and $\rho_{n}$ are eigenvalues of covariance matrix for $n=1,2, . . N$. The dimension of covariance matrix depends on $N$ which means that there is always $N-K$ repeated eigenvalues with $2 m_{i}-1$ repeated eigenvalues per band. This is expected since the derivation depends on the fact 
that all the bands depend on each other, thus (13) can be rewritten as

$$
\mu_{\gamma s}(s)=\prod_{i=1}^{K}\left(1-2 s\left(\log _{2}(M) S N R_{i} \cdot e_{i}\right)\right)^{-m_{i}}
$$

where $e$ is the eigenvalue of covariance matrix. We assume that the destination node is in the center of the node distribution and we try to calculate all the interference powers transmitting from all nodes by clustering the nodes into groups to determine the general formula for the total power interference.

In the $x^{\text {th }}$ order tier of the 3D distribution there are:

- The interference power at the destination node received from one of six nodes, at a distance $x d_{l}$, where $x$ represents the order of tier, is $\mu P_{t} /\left(d_{l} x\right)^{2}$.

- The interference power at the destination node received from one of eight nodes, at a distance $x \sqrt{3} d_{l}$, is $\mu P_{t} / 3\left(d_{l} x\right)^{2}$.

- The interference power at the destination node received from one of twelve nodes, at a distance $x \sqrt{2} d_{l}$, is $\mu P_{t} / 2\left(d_{l} x\right)^{2}$.

- The interference power at the destination node received from one of twenty nodes, at a distance $\sqrt{x^{2}+y^{2}} d_{l}$, where $\mathrm{y}=1, \ldots, \mathrm{x}-1$, and $x \geq 2$, is $\mu P_{t} / 2\left(d_{l} x\right)^{2}$.

- The interference power at the destination node received from one of twenty nodes, at a distance $\sqrt{2 x^{2}+y^{2}} d_{l}$, is $\mu P_{t} / d_{l}^{2}\left(x^{2}+y^{2}\right)$.

- The interference power at the destination node received from one of twenty nodes, at a distance $\sqrt{x^{2}+y^{2}+z^{2}} d$, where $\mathrm{z}=1, \ldots, \mathrm{x}-1$, and $x \geq 2$, is $\mu P_{t} / d_{l}^{2}\left(x^{2}+y^{2}+z^{2}\right)$.

A maximum $N$ and tier order $x^{\max }$ exist since the number of nodes in the network is finite. Therefore,

$$
\begin{aligned}
N & \approx \sum_{x=1}^{x_{\max }}(2 x+1)^{3}-(2(x-1)+1)^{3}, \\
& \approx \sum_{x=1}^{x_{\max }} 24 x^{2}+2=24 \frac{x_{\max }\left(x_{\max }+1\right)\left(2 x_{\max }+1\right)}{6}+2 x_{\max },
\end{aligned}
$$

For sufficiently large values of $N$ in $(17), x_{\max } \approx\left\lfloor(N)^{1 / 3} / 2\right\rfloor$. The probability of a single bit in the packet interfered by any node in the network is defined in $[8,11]$ as $1-\exp \left(-\lambda L / R_{b}\right)$ which means that the overall interference power using RESGO MAC protocol $\left(P_{I N I}{ }^{R E S G O}\right)$ can be expressed as [11]

$P_{I N I}{ }^{R E S G O}=\mu P_{t} \rho_{s}^{2 / 3}\left(1-e^{-\frac{\lambda L}{R}}\right) \times\left(\Delta_{1}+\Delta_{2}+\Delta_{3}-1\right)$,

where

$\Delta_{1}=\sum_{x=1}^{\frac{N^{1 / 3}}{2}} \frac{44}{3 x^{2}}$

$\Delta_{2}=\sum_{x=2}^{\frac{N^{1 / 3}}{2}} \sum_{y=1}^{x-1}\left(\frac{24}{2 x^{2}+y^{2}}+\frac{24}{x^{2}+y^{2}}\right)$

$$
\Delta_{3}=\sum_{x=2}^{\frac{N^{1 / 3}}{2}} \sum_{y=1}^{x-1} \sum_{z=1}^{y-1} \frac{24}{x^{2}+y^{2}+z^{2}}
$$

\section{NUMERICAL RESULTS}

The results in this section are obtained using the network simulation parameters that are indicated in the Table 1. Additionally, M-QAM modulation ( $\mathrm{M}=4$ and 16$)$, and $\mathrm{K}=3$ are considered.

Table.1 Simulation parameters

\begin{tabular}{|l|l|}
\hline Parameter & Value \\
\hline$G_{t}=G_{r}$ & 1 \\
\hline$f_{l}$ & $1.56 d B$ \\
\hline$F$ & $6 d B$ \\
\hline $\mathrm{V}$ & $1 \times 10^{6} \mathrm{~m}^{3}$ \\
\hline$\lambda L$ & $0.1 \mathrm{~b} / \mathrm{s}$ \\
\hline$P_{t}$ & $60 \mu \mathrm{w}$ \\
\hline $\mathrm{N}$ & 15000 \\
\hline
\end{tabular}

We derive the effective transport capacity for Nakagami-m fading channels in previous section. In order the numerical results to be comparable to the results in [11], we choose the value of $m=1$ for Nakagami-m fading channels, which represents Rayleigh fading channels. The effects of 3D node distribution on the effective transport capacity of adhoc dispersed spectrum cognitive radio networks are investigated through computer simulations considering $K=3$ dispersed channels between two nodes and the results are shown in Figs. 1 and 2. These two figures represent the relationship between the bit rate and the effective transport capacity considering 3D node distribution, different fading scenarios and modulation orders. It is shown that at low and high $R_{b}$ values, the effective transport capacity is low. However, at intermediate $R_{b}$ values, the effective transport capacity is saturated. This is due to the fact that the average sustainable number of hops is defined as the minimum between the maximum number of 
sustainable hops and the average number of hops per route. Full connectivity will not be sustained until reaching the average number of hops. Having reached the average number of hops, full connectivity will be sustained until the number of hops is greater than the threshold value as defined by an acceptable BER, since a low $S N R$ value is produced by low and high $R_{b}$ values.

It can be seen that the correlation between fading channels degrades the performance of the system and it can also be noted that configuration A case performs better than configuration $\mathrm{B}$ case. This is due to the fact that configuration $\mathrm{B}$ has higher correlation than that of configuration $\mathrm{A}$. In addition, the effects of modulation order on the effective transport capacity can be determined by comparison of Figs. 1 and 2. It is observed that the impact of modulation order on the effective transport capacity is negligible for independent and Configuration A cases whereas it is significant in case of Configuration B in which the channels are highly correlated. In Figs. 3, and 4 show the effective transport capacity when the nodes are distributed in $2 \mathrm{D}$. It is clearly noted that the distribution of 3D will decrease the effects of the INI, thus increasing the effective transport capacity.

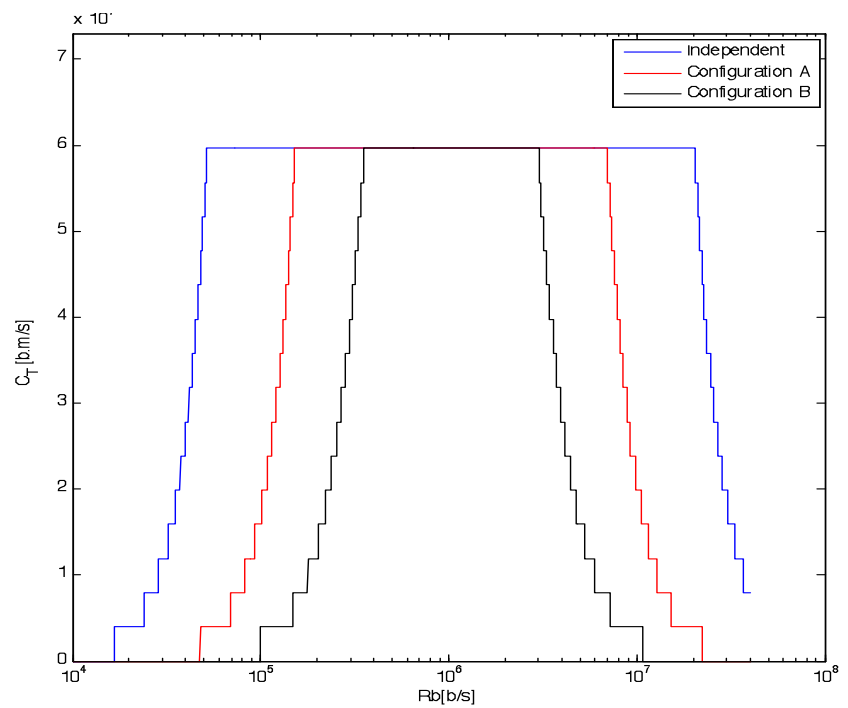

Fig.1 $C_{T}$ vs. $R_{b}$ for 3D node distribution considering $K=3$ Nakagami-m fading channels with $\mathrm{m}=1$ and 4-QAM modulation.

\section{CONCLUSION}

In this paper, we study the effects of the $3 \mathrm{D}$ node distribution along with INI on the effective transport capacity of adhoc dispersed spectrum cognitive radio networks. The effective transport capacity expressions are derived considering dependent and independent Nakagami-m fadings and different orders of M-QAM modulation schemes. The results show that effective transport capacity is saturated for intermediate bit rate values. Additionally, it is concluded that the correlation between fading channels highly affects the effective transport capacity. Last but not least, the modulation order has negligible and significant impacts on the effective transport capacity in the case of independent and slightly independent, and highly dependent fading channels, respectively. Note that this work can easily be extended to the case where each node transmits different power.

\section{ACKNOWLEDGEMENTS}

This work was supported in part by Qatar National Research Fund (QNRF) and in part by Qatar Telecom (Qtel).

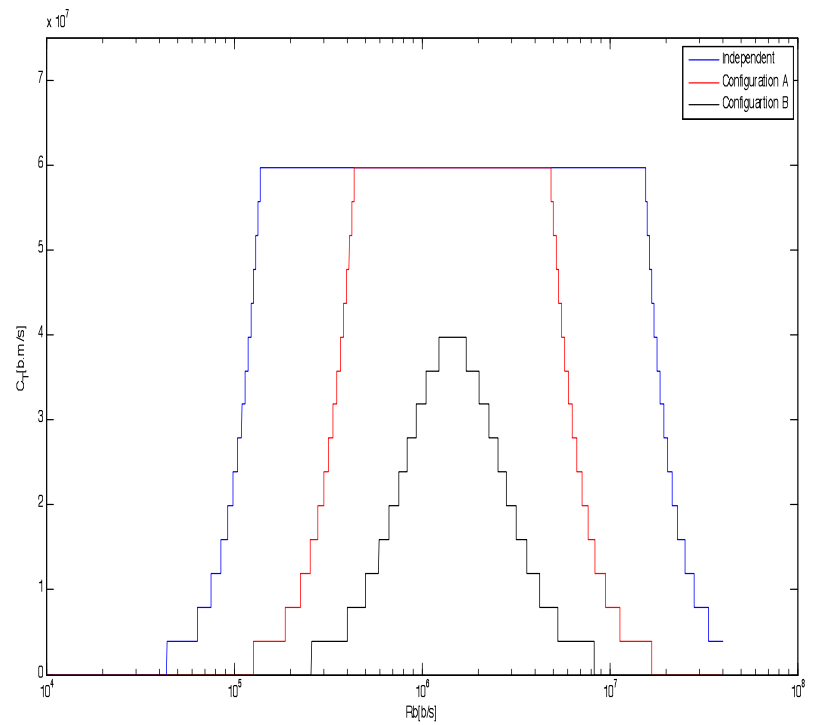

Fig. $2 C_{T}$ vs. $R_{b}$ for $3 \mathrm{D}$ node distribution considering $K=3$ Nakagami-m fading channels with $\mathrm{m}=1$ and 16-QAM modulation.

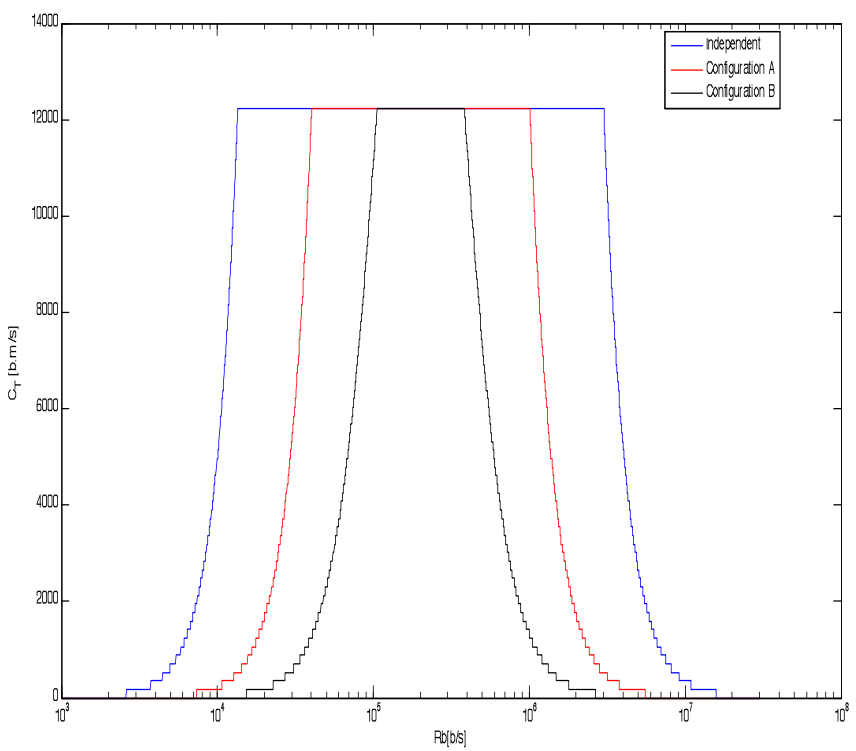

Fig.3 $C_{T}$ vs. $R_{b}$ for $2 \mathrm{D}$ node distribution with $\mathrm{K}=3$ Nakagami-m fading channels with $\mathrm{m}=1$ and 4-QAM modulation. 


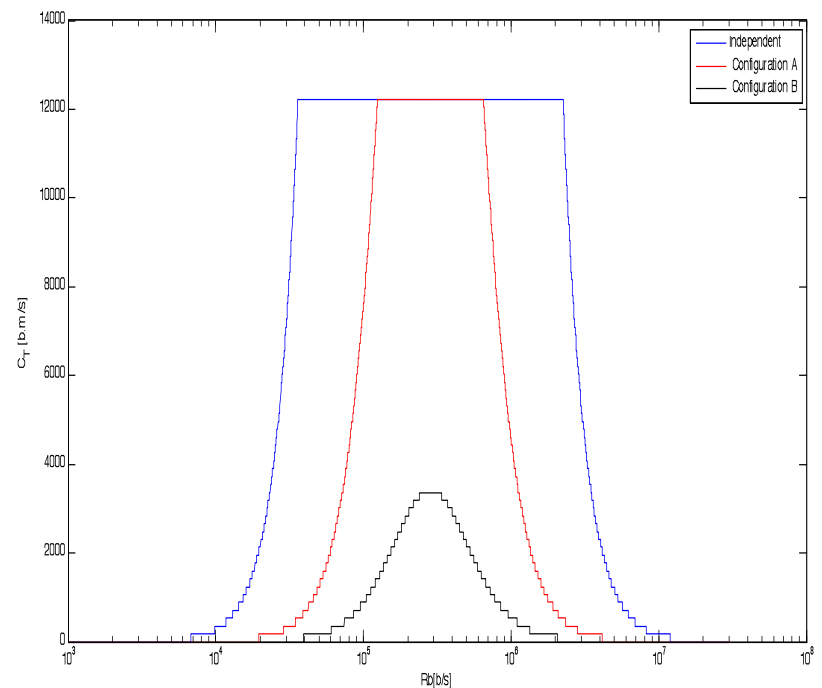

Fig.4 $C_{T}$ vs. $R_{b}$ for $2 \mathrm{D}$ node distribution with $K=3$ Nakagami-m fading channels with $\mathrm{m}=1$ and 16-QAM modulation.

\section{REFERENCES}

[1] C.E. Perkins, Ad Hoc Networking. Upper Saddle River, NJ, USA: Addison wesley, 2001.

[2] D. Pompili, T. Melodia, and I. Akyildiz, "Three-Dimensional and Two-dimensional Deployment Analysis of Underwater Acoustic SensorNetworks," Ad Hoc Networks (Elsevier), vol. 7, no. 4, pp. 778790, June 2009.

[3] J. Hoydis, M. Petrova, and P. Mähönen, "Effects of Topology on Local Throughput-Capacity of Ad Hoc Networks" Proc. of IEEE PIMRC 2008, Cannes, France, Sep. 2008.

[4] C. Liu and J. Wu, "Efficient Geometric Routing in Three Dimensional Ad Hoc Networks," Proc. of 27th Annual Joint Conf. of IEEE Communication and Computer Society (INFOCOM) (Miniconference), 2009.

[5] T. Chen, H. Zhang, G.M. Maggio and I. Chlamtac ,"Topology management in CogMesh: A cluster-based cognitive radio mesh network", Proc. IEEE ICC 2007 Workshop, Glasgow, UK, 2007.

[6] P. Mähönen, M. Petrova, and J. Riihijärvi, "Applications of topology information for cognitive radios and networks," New Frontiers in Dynamic Spectrum AccessNetworks, DySPAN 2007. 2nd IEEE International Symposium on, pp. 103-114, April, 2007.

[7] R. W. Thomas, R. S. Komali, L. A. DaSilva, and A. B. MacKenzie, "Joint Power and Channel Minimization in Topology Control: A Cognitive Network approach,"Proc. IEEE CogNet 2007 Workshop.

[8] O.K. Tonguz, and G. Ferrari, Ad Hoc Wireless Networks: A Communication Theoretic Perspective, New York: Wiley, 2006.

[9] G.Ferrari and O. K. Tonguz, "MAC protocols and transport capacity in ad hoc wireless networks: Aloha versus PR-CSMA," in Proc. IEEE Military Comm. Conf. (MILCOM), Boston, October 2003.

[10] S. Gezici, H. Celebi, H. V. Poor, and H. Arslan, "Fundamental limits on time delay estimation in dispersed spectrum cognitive radio systems," IEEE Trans. Wireless Commun., vol. 8, no. 1, pp. 78-83, Jan. 2009.

[11] G. Ferrari, B. Baruffini and O.K Tonguz, " Spectral efficiencyconnectivity tradeoff in ad hoc wireless networks," In proc. Intern, symp. On information theory and its applications (ISITA), Perma, Italy, Oct, 2004

[12] S.Haykin, Communication Systems, $3^{\text {rd }}$ ed, New York: Wiley, 1994.
[13] A. Goldsmith, Wireless Communciations, MIT: Cambridge Press, 2005

[14] M.k. Simon, and M-s. Alouini, Digital Communication Over Fading Channels A Unified Approach to Performance Analysis, Wiley, 2000.

[15] Nakagami, M.: 'The $\mathrm{m}$ distribution - a general formula forintensity distribution of rapid fading', in Hoffman, W.G. (Ed.): 'Statistical methods in radio wave propagation' (Pergamon, UK, 1960), pp. 3-36

[16] Q. Zhang," Exact analysis of Post detection combing for DPSK and NSFK systems over arbitrary correlated Nkagami-m channels," IEEE Trans. On Wireless Commun., Vol. 46, PP., 1459-1467, Nov., 1998.

[17] I. Ghareeb, "Noncoherent MT-MFSK signals with diversity reception arbitrarily correlated and unbalanced Nakagami-m fading channel.," IEEE Journ. on Select. Areas in Commun, Vol.23, pp.1839-1850, Sep 2005. 